\title{
Spheroid-based 3-dimensional culture models: Gene expression and functionality in head and neck cancer
}

\author{
MARIANNE SCHMIDT ${ }^{1}$, CLAUS-JUERGEN SCHOLZ ${ }^{2}$, CHRISTINE POLEDNIK ${ }^{1}$ and JEANETTE ROLLER ${ }^{1}$ \\ ${ }^{1}$ Department of Otorhinolaryngology, University of Würzburg, D-97080 Würzburg; \\ ${ }^{2}$ Interdisciplinary Center for Clinical Research, Microarray Core Unit, \\ University of Würzburg, D-97078 Würzburg, Germany
}

Received October 26, 2015; Accepted December 5, 2015

DOI: $10.3892 /$ or.2016.4581

\begin{abstract}
In the present study a panel of 12 head and neck cancer (HNSCC) cell lines were tested for spheroid formation. Since the size and morphology of spheroids is dependent on both cell adhesion and proliferation in the 3-dimensional (3D) context, morphology of HNSCC spheroids was related to expression of E-cadherin and the proliferation marker Ki67. In HNSCC cell lines the formation of tight regular spheroids was dependent on distinct E-cadherin expression levels in monolayer cultures, usually resulting in upregulation following aggregation into 3D structures. Cell lines expressing only low levels of E-cadherin in monolayers produced only loose cell clusters, frequently decreasing E-cadherin expression further upon aggregation. In these cell lines no epidermal growth factor receptor (EGFR) upregulation occurred and proliferation generally decreased in spheroids/aggregates independent of E-cadherin expression. In a second approach a global gene expression analysis of the larynx carcinoma cell line HLaC78 monolayer and the corresponding spheroids was performed. A global upregulation of gene expression in $\mathrm{HLaC} 78$ spheroids was related to genes involved in cell adhesion, cell junctions and cytochrome P450-mediated metabolism of xenobiotics. Downregulation was associated with genes controlling cell cycle, DNA-replication and DNA mismatch repair. Analyzing the expression of selected genes of each functional group in monolayer and spheroid cultures of all 12 cell lines revealed evidence for common gene expression shifts in genes controlling cell junctions, cell adhesion, cell cycle and DNA replication as well as genes involved in the cytochrome P450-mediated metabolism of xenobiotics.
\end{abstract}

Correspondence to: Dr Marianne Schmidt, Department of Otorhinolaryngology, University of Wuerzburg, Josef-SchneiderStrasse 11, D-97080 Wuerzburg, Germany

E-mail: schmidt_m2@ukw.de

Key words: cancer, carcinoma, head and neck, 3D culture, in vitro, HNSCC, E-cadherin, microarray, proliferation

\section{Introduction}

According to an analysis of over 3,000 cases of primary head and neck tumours in Germany in 2009, the patient benefit has not significantly improved from 1995 to 2006, despite new treatment strategies. Particularly, the 5-year overall survival rate for carcinomas of hypopharyngeal origin is very low with $27.2 \%$ (1). Hypopharyngeal cancer is often detected in later stages, since early signs and symptoms rarely occur. Late stages frequently involve multiple node affection, leading to poor prognosis (http://emedicine.medscape.com/article /1375268).

In vivo cells in a tissue context interact with neighbouring cells and extracellular matrix components. However, in 2-dimensional (2D) monolayer cultures cells tend to loose tissue-specific properties (2). 2D culture models therefore seem unsuitable to mimic the behaviour of cells in a natural, 3-dimensional (3D) microenvironment, particularly for testing of growth or invasion inhibiting substances, just as proposed in 1988 (3). In many aspects spheroids resemble the solid, nonvascularized tumour tissue and spheroidal cells show higher morphological and functional differentiation than monolayer cells. Upon proliferation of cells inside spheroids a tumourlike microenvironment develops with a hypoxic/necrotic core (reviewed in ref. 4), surrounded by quiescent and proliferating zones. Cells inside spheroids secrete extracellular matrix and functional cell junctions arise between cells, providing an advanced model system for examination of growth and invasion of carcinomas (4).

In the present study we compared the morphology and size of $12 \mathrm{HNSCC}$ cell lines with the expression of the proliferation marker Ki67 and the cell-cell adhesion marker E-cadherin. Furthermore, the larynx carcinoma cell line HLaC78 was used as a model for comparing overall gene expression in 2D monolayer cultures with $72 \mathrm{~h}$ old HLaC78 multicellular spheroids. The results provide evidence for extensive restructuring of gene expression in the 3D context.

\section{Materials and methods}

Cell lines and cell culture. The cell lines used for spheroid analysis are listed in Table I. The cell lines were kept in RPMI1640, 10\% FCS and standard antibiotics, except Hep2 (MEM), 
Table I. HNSCC cell lines used in the present study.

\begin{tabular}{ll}
\hline Cell line & \multicolumn{1}{c}{ Origin } \\
\hline FADU & Hypopharynx carcinoma, LGC standards (Wesel, Germany) \\
HLaC78 & Larynx carcinoma (5) \\
Hep-2 & Larynx carcinoma , LGC standards (Wesel, Germany) \\
Hep-2-Tax & Larynx carcinoma, paclitaxel-resistant clone \\
HLaC79 & Larynx carcinoma (5) \\
HLaC79-Tax & Larynx carcinoma, paclitaxel-resistant clone \\
HPaC79 & Parotis carcinoma (6) \\
HSmC78 & Submandibular gland (5) \\
Cal27 & Tongue, LGC standards (Wesel, Germany) \\
PE/CA-PJ41 & Tongue, Sigma-Aldrich (Taufkirchen, Germany) (7) \\
SCC4 & Tongue, LGC standards (Wesel, Germany) \\
HNO210 & Larynx carcinoma, CLS (Eppelheim, Germany)
\end{tabular}

${ }^{\text {aS }}$ upposed to be HeLa contaminated, http://www.lgcstandards-atcc.org/products/all/CCL-23. HNSCC, head and neck cancer.

Hep2-Tax (MEM+20 $\mu \mathrm{M}$ paclitaxel), SCC4 [Dulbecco's modified Eagle's medium (DMEM/F12)] and HNO 210 (DMEM).

Spheroid formation. Tumour spheroids were generated by seeding 5,000 cells/well of Hep2 and Hep2-Tax cells in ultra-low attachment (ULA) 96-well round-bottomed plates (Corning, Amsterdam, The Netherlands) (8) or alternatively in ULA T75 flasks. Images were captured after $72 \mathrm{~h}$ with a Leica DMI 4000 inverted fluorescence microscope (Leica Microsystems, Wetzlar, Germany).

\section{Expression micro array}

RNA extraction and RNA quality control. RNA of HLaC78 monolayer and spheroidal cell cultures was isolated with the RNeasy kit (Qiagen, Hilden, Germany) according to the manufacturer's instructions. RNA quality was assessed with the RNA 6000 Nano kit using the Bioanalyzer 2100 instrument (Agilent, Böblingen, Germany). RNA integrity numbers (RINs) of our samples ranged between 9.4 and 9.9.

Microarray analysis. For microarray hybridization, $100 \mathrm{ng}$ total RNA were amplified and labelled using the IVT Express kit and hybridized to GeneChip PrimeView Human Gene Expression arrays (both from Affymetrix, Santa Clara, CA, USA) according to the manufacturer's instructions.

Raw microarray data was background corrected, normalized and summarized to probe set expression values using the robust microarray average (RMA) [algorithm $(9,10)$ ]. Data preprocessing and calculation of $\log _{2}$ fold-changes between spheroid and monolayer expression values was performed in the $\mathrm{R}$ environment (http://www.r-project.org) using the Bioconductor (http://www.bioconductor.org) package 'affy'. Pathway enrichment analyses were performed with gene set enrichment analysis (GSEA) (11). Microarray data were deposited in MIAME-compliant form at Gene Expression Omnibus (http://www.ncbi.nlm.nih.gov/geo) with the identifier GSE67495.
Real-time PCR. Gene expression rates were determined by real-time TaqMan ${ }^{\circledR}$ PCR (Applied Biosystems, Foster City, CA, USA). RNA was isolated from cell lines and primary cells with the RNeasy kit according to the manufacturer's instructions. The High Capacity RNA-to-cDNA Master Mix (Applied Biosystems, Darmstadt, Germany) was used for cDNA reverse transcription. Real-time PCR was performed in triplicates on a Real-time PCR cycler (Applied Biosytems, Darmstadt, Germany) using the TaqMan ${ }^{\circledR}$ gene expression assays for E-cadherin (CDH1), N-cadherin (CDH2), Ki67 (MKI), gap junction protein $\beta 6$ (GJB6), aldo-keto reductase family 1 member C1 (AKR1C1), cytochrome P450 1B1 (CYP1b1), flap endonuclease 1 (FEN1), mutL homolog 1 (MLH1), [replication factor C (activator 1) 3 (RFC3)], and epidermal growth factor receptor 1 (EGFR1).

Relative quantification was calculated according to the $2^{-\Delta \Delta \mathrm{Ct}}$ method (12). Expression values were normalized to the expression of GAPDH as an endogenous control which proved to be expressed most stably throughout the cell lines.

Scanning electron microscopy. For examination of morphology of HLaC78 spheroidal surface SEM was performed. Spheroids were fixed overnight at $4^{\circ} \mathrm{C}$ with $2 \%$ glutaraldehyde in $0.1 \mathrm{M}$ phosphate-buffer. Subsequently, spheroids were treated with $2 \%$ osmiumtetroxide in $0.1 \mathrm{M}$ phosphate-saccharose. After dehydration in graded ethanol series (35-100\%), specimens were dried using a critical point dryer. Spheroids were then coated with carbon, sputtered with gold palladium particles and viewed with an SEM (DSM 962; Zeiss, Oberkochen, Germany).

Statistical analysis. All statistical analyses and graphs were performed with GraphPad Prism 4 (GraphPad Software, Inc., La Jolla, CA, USA).

\section{Results}

Morphology of spheroids and expression of crucial genes. Cells $(5,000)$ of each cell line (Table I) were seeded into 


\section{Tight}
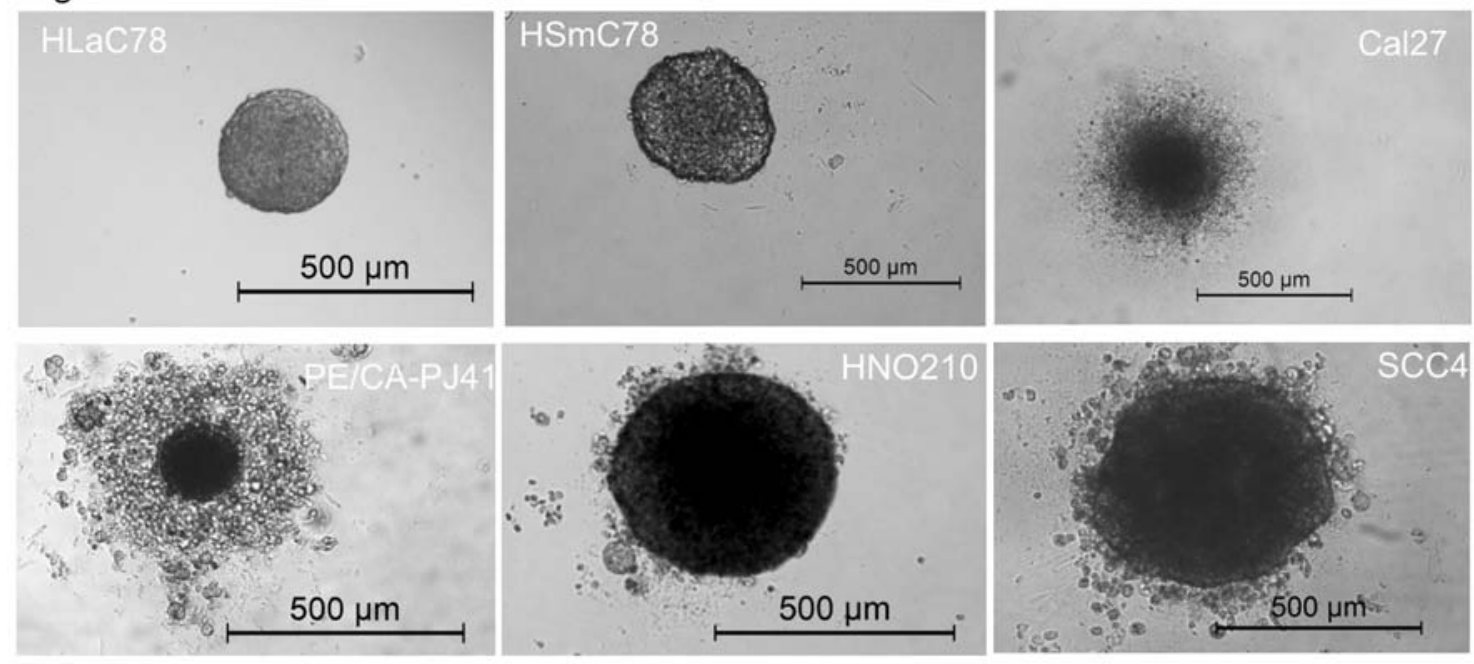

\section{Intermediate}
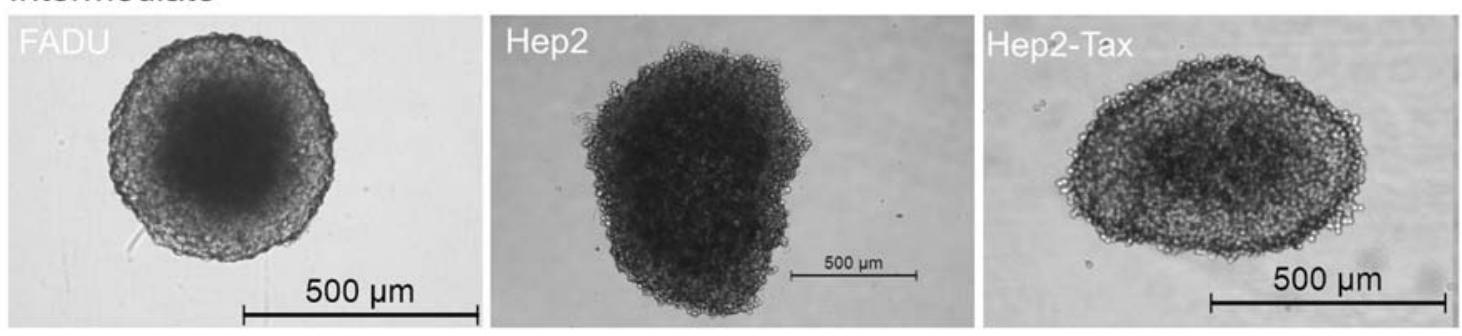

\section{Loose}
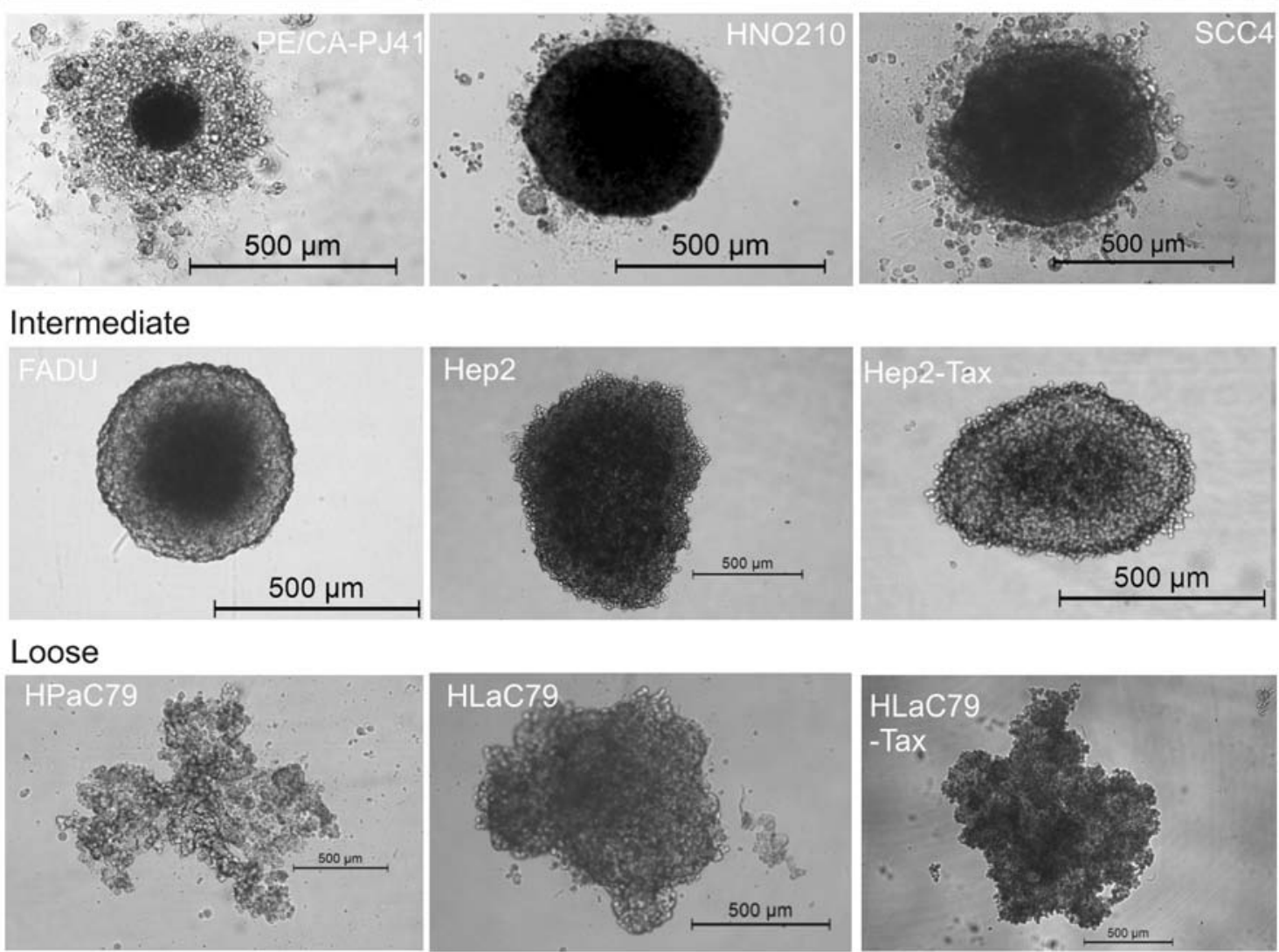

Figure 1. Spheroids of 10 different HNSCC cell lines established from 5,000 cells in ULA 96-well plates with conical bottom $72 \mathrm{~h}$ after seeding. Spheroids were grouped according to tightness.

96 ULA U-bottom well plates. Fig. 1 shows $72 \mathrm{~h}$ old spheroids originating from 5,000 cells of each cell line.

Fig. 1 demonstrates the morphological differences between spheroids of different monolayer origin. While HLaC78 and HSmC78 form small and very tight, nearly indestructible spheroids (even by harsh pipetting upon trypsin incubation), FADU, HNO210 and SCC-4 spheroids are larger in diameter, but also round shaped. Cal27 and PE/ CA-PJ41 are typically surrounded by a halo of dead/nonaggregated cells. HPaC79, Hep2/Hep2-Tax and HLaC79/ HLaC79-Tax cell lines rather aggregate into loose clusters. Spheroid size ranges from $\sim 250 \mu \mathrm{m}$ for tight, compact spheroids (HLaC78 and HsmC78) till diameters $>1 \mathrm{~mm}$ (HPaC79).

In general spheroid sizes are both determined by tightness of cell-cell contacts and proliferation rates of the cell lines within the aggregates. Tightness of the spheroids and the proliferation rates within were measured by qRT-PCR expression analysis of E-cadherin (as a marker for adhesion) and Ki67 (as a proliferation marker; Fig. 2) and opposed to expression of these markers in monolayers.

It is noticeable, that tight spheroids show either strong upregulation of E-cadherin upon aggregation (as HLaC78, zHSmC78 Cal27 or Pe/CA-PJ41). or a basic high E-cadherin expression in monolayer cultures with moderate increase/decrease (HNO210 and SSC4) following spheroid formation. Cell lines, agglomerating into intermediate or loose spheroids featured a basically low E-cadherin expression, increasing only weakly or decreasing upon loss of attachment to 2D surfaces (Fig. 2). Proliferation decreased in all cell lines except FADU, most markedly in cell lines forming tight spheroids. Since usually E-cadherin expression causes a decrease in proliferation (13) it is noticeable that the cell lines HPaC79, Hep2 and Hep2-Tax on the one hand decreased E-cadherin expression upon spheroid formation (log foldchanges HPaC79 -1.06, Hep2 -1.62, Hep2-Tax -2.20 and on the other hand also proliferation diminished (log fold-changes Ki67: HPaC79 -4.02, Hep2 -0.48 and Hep2-Tax -1.44; see 


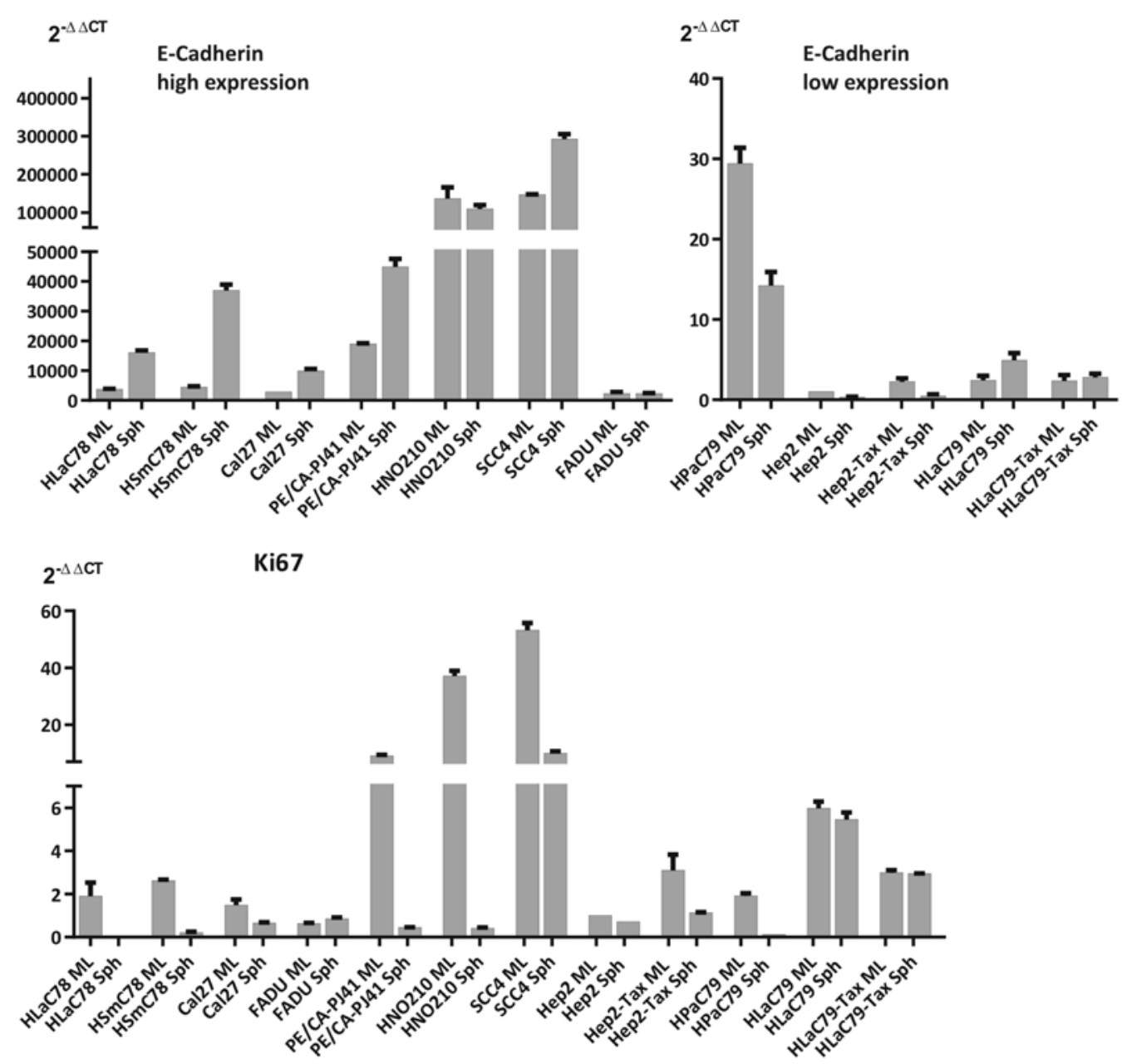

Figure 2. Expression of E-cadherin and Ki67 in monolayer cultures compared to spheroids of 12 different HNSCC cell line. For adequate presentation of E-cadherin cell lines were divided into groups expressing high or low levels of E-cadherin in monolayer cultures.

Table IV). Wang et al (14) described an enhancement of proliferation by downregulation of E-cadherin in HNSCC monolayer cell lines. The enhanced proliferation was mediated by upregulation of EGFR. To check this in the cell lines HPaC79, Hep2 and Hep2-Tax we performed qRT-PCR for EGFR. In these cell lines E-cadherin was downregulated along with EGFR in Hep2 (EGFR log fold-change -0.90) and Hep2-Tax (EGFR log fold-change 1.35) following aggregation. $\mathrm{HPaC} 79$ did not express EGFR in monolayer or in the spheroid culture.

Gene expression. Microarray analysis was used to create a gene expression array profile of head and neck cancer cell line growing as monolayer and in a 3D context. We selected the HLaC78 cell line due to its spontaneous aggregation into tight, well-shaped spheroids with a smooth, tissue-like surface with apical microvilli protrusions typical for epithelia (Fig. 3). A global upregulation of gene expression in HLaC78 larynx carcinoma cells line growing in a tissue-like 3D structure was primarily related to cell adhesion, cell junctions and cytochrome P450-mediated metabolism of xenobiotics. Expression downregulation could be predominantly associated with cell cycle, DNA replication and DNA mismatch repair.

A summary of selected differentially expressed probe sets is shown in Table II, subdivided into functional groups.
To transfer data obtained from HLaC78 expression analysis and to determine key changes in switching between 2D and 3D conditions, the expression of selected genes of each functional group were analyzed by quantitative RT-PCR in monolayer and spheroid cultures of 12 different HNSCC cell lines.

Genes selected for TaqMan ${ }^{\circledR}$ qRT-PCR expression quantification in monolayer and spheroid cultures of HNSCC cell lines are summarized in Table III, log fold-changes for spheroids of each cell line in comparison with monolayer culture are shown in Table IV.

Common expression shifts occurred in cell lines forming tight spheroids under 3D conditions. Thus, expression of cell junction and cell adhesion genes, as well as cytochrome P450 detoxification genes increased (GJB6, E-cadherin, AKR1C1, Cyp1a1 and 1b1), while expression of genes important for proliferation and DNA-replication (MKI and RCF3) decreased. In the DNA mismatch repair group no common tendency of expression shift was visibleX (at least for the tested FEN1/MLH1 genes). In cell lines, forming only loose aggregates, no upregulation of GJB6 or E-cadherin occurred under 3-dimensional conditions. Cell proliferation and DNA replication genes, however, were downregulated independent of spheroid compaction in the 3D context. Notably, the tested DNA mismatch repair genes were uniformly downregulated in cell lines aggregating loosely. 
Table II. Log fold-changes in gene expression in spheroids vs. monolayer cultures of HLaC78 cell line.

\begin{tabular}{|c|c|c|}
\hline Gene symbol & Gene & $\operatorname{logFC}$ \\
\hline \multicolumn{3}{|l|}{ Cell junctions } \\
\hline GJB6 & Gap junction protein, $\beta 6,30 \mathrm{kDa}$ & 4,588 \\
\hline $\mathrm{CDH} 2$ & Cadherin 2 , type $1, \mathrm{~N}$-cadherin (neuronal) & $-3,963$ \\
\hline$G J B 2$ & Gap junction protein, $\beta 2,26 \mathrm{kDa}$ & 3,547 \\
\hline CLDN10 & Claudin 10 & 3,364 \\
\hline$D S C 2$ & Desmocollin 2 & 3,254 \\
\hline$D S G 3$ & Desmoglein 3 & 2,727 \\
\hline NOTCH3 & Notch 3 & 2,723 \\
\hline GJAl & Gap junction protein, $\alpha 1,43 \mathrm{kDa}$ & 2,488 \\
\hline DSC3 & Desmocollin 3 & 2,238 \\
\hline$C L D N 7$ & Claudin 7 & 2,222 \\
\hline NOTCH1 & Notch 1 & 1,991 \\
\hline$F 11 R$ & F11 receptor & 1,887 \\
\hline CLDN8 & Claudin 8 & 1,878 \\
\hline$C A V 1$ & Caveolin 1 , caveolae protein, $22 \mathrm{kDa}$ & $-1,730$ \\
\hline$J U P$ & Junction plakoglobin & 1,657 \\
\hline $\mathrm{CDHI}$ & Cadherin 1, type 1, E-cadherin (epithelial) & 1,589 \\
\hline$I T G B 1$ & Integrin, $\beta 1$ (fibronectin receptor) & $-1,195$ \\
\hline$D S P$ & Desmoplakin & 1,181 \\
\hline ITGB6 & Integrin, $\beta 6$ & 1,099 \\
\hline GJB5 & Gap junction protein, $\beta 5,31.1 \mathrm{kDa}$ & 1,041 \\
\hline \multicolumn{3}{|l|}{ Cell adhesion } \\
\hline$H L A-D R A$ & Major histocompatibility complex, class II, DR $\alpha$ & 7,514 \\
\hline$H L A-D R B 1$ & Major histocompatibility complex, class II, DR $\beta 1$ & 4,842 \\
\hline$H L A-D M A$ & Major histocompatibility complex, class II, DM $\alpha$ & 4,692 \\
\hline HLA-DPA1 & Major histocompatibility complex, class II, DP $\alpha 1$ & 4,391 \\
\hline$H L A-F$ & Major histocompatibility complex, class I, F & 4,050 \\
\hline$H L A-D M B$ & Major histocompatibility complex, class II, D $\beta$ & 3,924 \\
\hline$H L A-D R B 5$ & Major histocompatibility complex, class II, DR $\beta 5$ & 3,864 \\
\hline$H L A-D P B 1$ & Major histocompatibility complex, class II, DP $\beta 1$ & 3,464 \\
\hline CLDN10 & Claudin 10 & 3,364 \\
\hline$H L A-B$ & Major histocompatibility complex, class I, B & 2,965 \\
\hline$H L A-C$ & Major histocompatibility complex, class I, C & 2,618 \\
\hline$H L A-D Q B 1$ & Major histocompatibility complex, class II, DQ $\beta 1$ & 2,477 \\
\hline$H L A-E$ & Major histocompatibility complex, class I, E & 2,468 \\
\hline$C L D N 7$ & Claudin 7 & 2,222 \\
\hline PTPRM & Protein tyrosine phosphatase, receptor type, $\mathrm{M}$ & 2,192 \\
\hline CD58 & CD58 molecule & 2,076 \\
\hline$H L A-A$ & Major histocompatibility complex, class I, A & 2,058 \\
\hline PVRL1 & Poliovirus receptor-related 1 (herpesvirus entry mediator C) & 2,016 \\
\hline$F 11 R$ & F11 receptor & 1,887 \\
\hline$C L D N 8$ & Claudin 8 & 1,878 \\
\hline $\mathrm{CDH}$ & Cadherin 1, type 1, E-cadherin (epithelial) & 1,589 \\
\hline $\mathrm{CDH} 3$ & Cadherin 3, type 1, P-cadherin (placental) & 1,566 \\
\hline NEO1 & Neogenin 1 & 1,518 \\
\hline CD86 & CD86 molecule & 1,424 \\
\hline$I T G B 8$ & Integrin, $\beta 8$ & 1,370 \\
\hline GLG1 & Golgi glycoprotein 1 & 1,368 \\
\hline CNTN1 & Contactin 1 & 1,366 \\
\hline$C D 40$ & CD40 molecule, TNF receptor superfamily member 5 & 1,359 \\
\hline$S D C 1$ & Syndecan 1 & 1,340 \\
\hline$S D C 2$ & Syndecan 2 & 1,265 \\
\hline PTPRF & Protein tyrosine phosphatase, receptor type, $\mathrm{F}$ & 1,159 \\
\hline$C D 276$ & CD276 molecule & 1,100 \\
\hline
\end{tabular}


Table II. Continued.

\begin{tabular}{|c|c|c|}
\hline Gene symbol & Gene & $\operatorname{logFC}$ \\
\hline \multicolumn{3}{|c|}{ Cytochrome P450 metabolism } \\
\hline AKRICl & Aldo-keto reductase family 1 , member $\mathrm{C} 1$ & 3,798 \\
\hline$A K R 1 C 3$ & Aldo-keto reductase family 1 , member $\mathrm{C} 3$ & 3,364 \\
\hline CYP1B1 & Cytochrome P450, family 1 , subfamily B, polypeptide 1 & 2,837 \\
\hline CYPIAl & Cytochrome P450, family 1 , subfamily A, polypeptide 1 & 2,327 \\
\hline GSTA4 & Glutathione S-transferase $\alpha 4$ & 2,299 \\
\hline$A L D H 3 B 2$ & Aldehyde dehydrogenase 3 family, member B2 & 2,039 \\
\hline MGST2 & Microsomal glutathione S-transferase 2 & 1,196 \\
\hline GSTK1 & Glutathione S-transferase $\kappa 1$ & 1,131 \\
\hline GSTZ1 & Glutathione S-transferase $\zeta 1$ & 1,112 \\
\hline \multicolumn{3}{|l|}{ Cell cycle } \\
\hline$C D K N 3$ & Cyclin-dependent kinase inhibitor 3 & $-5,795$ \\
\hline$C D K 1$ & Cyclin-dependent kinase 1 & $-5,556$ \\
\hline BIRC5 & Baculoviral IAP repeat containing 5 & $-5,257$ \\
\hline CCNA2 & Cyclin A2 & $-5,205$ \\
\hline$C D C 20$ & Cell division cycle 20 homolog (S. cerevisiae) & $-5,19$ \\
\hline CDC6 & Cell division cycle 6 homolog (S. cerevisiae) & $-5,104$ \\
\hline$M A D 2 L 1$ & MAD2 mitotic arrest deficient-like 1 (yeast) & $-4,665$ \\
\hline CCNB1 & Cyclin B1 & $-4,573$ \\
\hline$C D C 25 A$ & Cell division cycle 25 homolog A (S.pombe) & $-4,500$ \\
\hline$A U R K B$ & Aurora kinase B & $-4,439$ \\
\hline$C C N B 2$ & Cyclin B2 & $-4,387$ \\
\hline MKI67 & Antigen identified by monoclonal antibody Ki67 & $-4,028$ \\
\hline BRCAl & Breast cancer 1 , early onset & $-3,798$ \\
\hline RAD51 & RAD51 homolog (S.cerevisiae) & $-3,543$ \\
\hline KNTC1 & Kinetochore associated 1 & $-3,023$ \\
\hline MCM3 & Minichromosome maintenance complex component 3 & $-2,973$ \\
\hline CKS2 & CDC28 protein kinase regulatory subunit 2 & $-2,919$ \\
\hline STMN1 & Stathmin 1 & $-2,783$ \\
\hline$A U R K A$ & Aurora kinase A & $-2,778$ \\
\hline GTSE1 & G-2 and S-phase expressed 1 & $-2,719$ \\
\hline CHEK1 & Checkpoint kinase 1 & $-2,687$ \\
\hline$C K S 1 B$ & $\mathrm{CDC} 28$ protein kinase regulatory subunit $1 \mathrm{~B}$ & $-2,636$ \\
\hline$C C N D 2$ & Cyclin D2 & $-2,488$ \\
\hline TFDP1 & Transcription factor Dp-1 & $-2,242$ \\
\hline BCCIP & BRCA2 and CDKN1A interacting protein & $-2,239$ \\
\hline TFDP1 & Transcription factor Dp-1 & $-2,219$ \\
\hline$B R C A 2$ & Breast cancer 2 , early onset & $-2,147$ \\
\hline$C D K N 1 A$ & Cyclin-dependent kinase inhibitor 1A (p21, Cip1) & 2,116 \\
\hline$C C N F$ & Cyclin F & $-2,103$ \\
\hline$C D K 2$ & Cyclin-dependent kinase 2 & $-1,949$ \\
\hline$R B L 1$ & Retinoblastoma-like 1 (p107) & $-1,905$ \\
\hline$S K P 2$ & S-phase kinase-associated protein 2, E3 ubiquitin protein ligase & $-1,580$ \\
\hline$M D M 2$ & Mdm2, p53 E3 ubiquitin protein ligase homolog (mouse) & 1,454 \\
\hline$R A D 1$ & RAD1 homolog (S.pombe) & $-1,416$ \\
\hline CCNE1 & Cyclin E1 & $-1,379$ \\
\hline CCNC & Cyclin C & $-1,336$ \\
\hline$M A D 2 L 2$ & MAD2 mitotic arrest deficient-like 2 (yeast) & $-1,263$ \\
\hline$N B N$ & Nibrin & $-1,249$ \\
\hline$C D K 4$ & Cyclin-dependent kinase 4 & $-1,245$ \\
\hline$R B 1$ & Retinoblastoma 1 & $-1,219$ \\
\hline MCM4 & Minichromosome maintenance complex component 4 & $-1,203$ \\
\hline STMN1 & Stathmin 1 & $-1,193$ \\
\hline$C D K 5 R 1$ & Cyclin-dependent kinase 5, regulatory subunit 1 (p35) & $-1,177$ \\
\hline
\end{tabular}


Table II. Continued.

\begin{tabular}{|c|c|c|}
\hline Gene symbol & Gene & $\log \mathrm{FC}$ \\
\hline CCNG2 & Cyclin G2 & 1,102 \\
\hline CDK6 & Cyclin-dependent kinase 6 & $-1,094$ \\
\hline BCCIP & BRCA2 and CDKN1A interacting protein & $-1,085$ \\
\hline$C C N D 1$ & Cyclin D1 & $-1,064$ \\
\hline \multicolumn{3}{|c|}{ DNA mismatch repair } \\
\hline FEN1 & Flap structure-specific endonuclease 1 & $-2,869$ \\
\hline NEIL3 & Nei endonuclease VIII-like 3 (E. coli) & $-2,787$ \\
\hline POLE2 & Polymerase (DNA directed), $\varepsilon 2$, accessory subunit & $-2,760$ \\
\hline PCNA & Proliferating cell nuclear antigen & $-2,279$ \\
\hline$H M G B 1$ & High mobility group box 1 & $-2,076$ \\
\hline PARP1 & Poly(ADP-ribose) polymerase 1 & $-1,931$ \\
\hline$L I G 1$ & Ligase I, DNA, ATP-dependent & $-1,724$ \\
\hline POLE & Polymerase (DNA directed), $\varepsilon$, catalytic subunit & $-1,621$ \\
\hline POLD3 & Polymerase (DNA-directed), $\delta 3$, accessory subunit & $-1,387$ \\
\hline$M B D 4$ & Methyl-CpG binding domain protein 4 & $-1,378$ \\
\hline$M L H 1$ & mutL homolog 1 , colon cancer, non-polyposis type 2 (E. coli) & $-1,369$ \\
\hline POLD2 & Polymerase (DNA directed), $\delta 2$, accessory subunit & $-1,248$ \\
\hline POLD1 & Polymerase (DNA directed), $\delta 1$, catalytic subunit & $-1,182$ \\
\hline NEIL2 & Nei endonuclease VIII-like 2 (E. coli) & $-1,143$ \\
\hline$U N G$ & Uracil-DNA glycosylase & $-1,132$ \\
\hline \multicolumn{3}{|c|}{ DNA replication } \\
\hline RFC3 & Replication factor C (activator 1) 3, $38 \mathrm{kDa}$ & $-3,710$ \\
\hline POLA2 & Polymerase (DNA directed), $\alpha 2$, accessory subunit & $-3,076$ \\
\hline$M C M 3$ & Minichromosome maintenance complex component 3 & $-2,973$ \\
\hline PRIM1 & Primase, DNA, polypeptide 1 (49 kDa) & $-2,948$ \\
\hline FEN1 & Flap structure-specific endonuclease 1 & $-2,869$ \\
\hline MCM6 & Minichromosome maintenance complex component 6 & $-2,859$ \\
\hline$M C M 7$ & Minichromosome maintenance complex component 7 & $-2,853$ \\
\hline POLE2 & Polymerase (DNA directed), $\varepsilon 2$, accessory subunit & $-2,760$ \\
\hline MCM4 & Minichromosome maintenance complex component 4 & $-2,616$ \\
\hline DNA2 & DNA replication helicase/nuclease 2 & $-2,429$ \\
\hline RNASEH $2 A$ & Ribonuclease $\mathrm{H} 2$, subunit $\mathrm{A}$ & $-2,381$ \\
\hline$M C M 2$ & Minichromosome maintenance complex component 2 & $-2,344$ \\
\hline PCNA & Proliferating cell nuclear antigen & $-2,279$ \\
\hline RFC5 & Replication factor C (activator 1) $5,36.5 \mathrm{kDa}$ & $-2,227$ \\
\hline RFC4 & Replication factor C (activator 1 ) $4,37 \mathrm{kDa}$ & $-2,086$ \\
\hline$R F C 2$ & Replication factor C (activator 1) 2, $40 \mathrm{kDa}$ & $-1,798$ \\
\hline$L I G 1$ & Ligase I, DNA, ATP-dependent & $-1,724$ \\
\hline POLAl & Polymerase (DNA directed), $\alpha 1$, catalytic subunit & $-1,671$ \\
\hline MCM5 & Minichromosome maintenance complex component 5 & $-1,622$ \\
\hline POLE & Polymerase (DNA directed), $\varepsilon$, catalytic subunit & $-1,621$ \\
\hline POLD3 & Polymerase (DNA-directed), $\delta 3$, accessory subunit & $-1,387$ \\
\hline$R P A 3$ & Replication protein $\mathrm{A} 3,14 \mathrm{kDa}$ & $-1,368$ \\
\hline RNASEH2B & Ribonuclease $\mathrm{H} 2$, subunit B & $-1,260$ \\
\hline POLD2 & Polymerase (DNA directed), $\delta 2$, accessory subunit & $-1,248$ \\
\hline RFCl & Replication factor C (activator 1) $1,145 \mathrm{kDa}$ & $-1,235$ \\
\hline PRIM2 & Primase, DNA, polypeptide 2 (58 kDa) & $-1,185$ \\
\hline POLD1 & Polymerase (DNA directed), $\delta 1$, catalytic subunit & $-1,182$ \\
\hline POLE3 & Polymerase (DNA directed), $\varepsilon 3$, accessory subunit & $-1,123$ \\
\hline
\end{tabular}



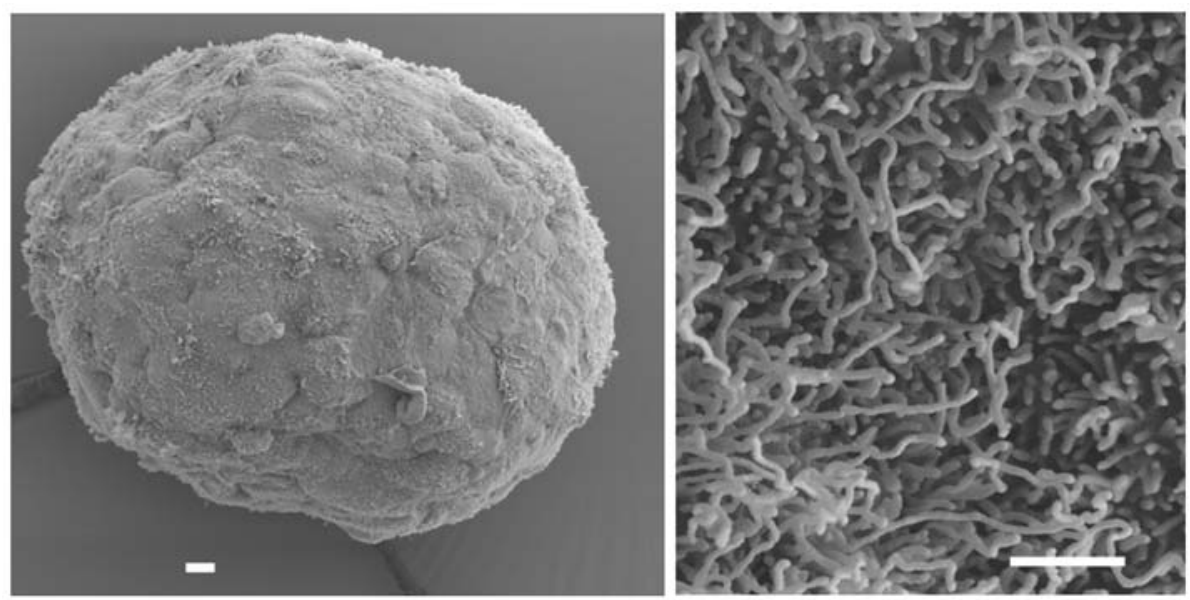

Figure 3. Scanning electron microscopy (SEM) display of a typical HLaC78 spheroid surface. Bar, $1 \mu \mathrm{m}$.

Table III. Genes tested for expression in monolayer and spheroid cultures of different HNSCC cell lines.

\begin{tabular}{|c|c|}
\hline Functional group & Gene \\
\hline Cell junctions & GJB6, gap junction protein $\beta 6$ \\
\hline Cell adhesion & CDH1, E-cadherin \\
\hline \multirow[t]{3}{*}{$\begin{array}{l}\text { Cytochrome P450 } \\
\text { metabolism }\end{array}$} & $\begin{array}{l}\text { AKR1C1, aldo-keto family } 1 \\
\text { reductase member }\end{array}$ \\
\hline & CYP1B1, cytochrome P450 1B1 \\
\hline & CYP1A1, cytochrome P450 1A1 \\
\hline Cell cycle & MKI, Ki67 proliferative antigen \\
\hline DNA mismatch repair & FEN1, flap endonuclease 1 \\
\hline DNA replication & $\mathbf{R F C 3}$, replication factor $\mathrm{C} 3$ \\
\hline
\end{tabular}

HNSCC, head and neck cancer.

\section{Discussion}

The present study provides an overview of spheroid formation properties of 12 HNSCC cell lines. Spheroids differed enormously in size and tightness. The cell lines were divided in those forming tight spheroids or intermediate/ loose aggregates. Since the size of spheroids is in general dictated by cell adhesion and proliferation rates within spheroids, expression profiles of Ki67 (proliferation) and E-cadherin (adhesion) were generated. For generation of tight, regular spheroids a distinct level of E-cadherin expression in monolayers was necessary and underwent a further significant upregulation upon aggregation. Alternatively, this further significant upregulation seemed to be expendable, when E-cadherin expression in monolayer cell lines was exceptionally high as in $\mathrm{HNO} 210$ or SCC4 cell lines, respectively. The cell lines expressing only low levels of E-cadherin under two-dimensional (2D) conditions were not able to gain a sufficient increase in E-cadherin expression to generate compact spheroidal tissue-like aggregates. Most of these cell lines even decreased E-cadherin expression under non-adherent conditions. Proliferation, however, decreased in all cell lines (except FADU) when the attachment to cell culture surfaces was suspended and 3-dimensional growth was initiated. The observation that spheroid tightness in HNSCC is partially determined by the expression of E-cadherin in monolayer culture is in agreement with the observation in other systems (15). In general upregulation of E-cadherin causes a $\beta$-catenin-ediated contact inhibition and subsequent decrease in proliferation (13). Furthermore, it has been shown that knock-down of E-cadherin enhances the proliferation of HNSCC cell lines by upregulating epidermal growth factor (EGFR) receptor. Despite the usual pathway of E-cadherinmediated contact inhibition, in the cell lines HPaC78, Hep2 and Hep2-Tax, all forming only loose aggregates, E-cadherin was downregulated, but yet proliferation decreased. Obviously pathways initiated in monolayer cell cultures are not uniformly conferrable to the $3 \mathrm{D}$ context.

A comparison of overall gene expression between the monolayer culture of the human larynx carcinoma cell line HLaC78 and the corresponding $72 \mathrm{~h}$ old spheroids was performed. HLaC78 cells form tight spheroids spontaneously with a smooth, ciliated surface. Gene expression changed towards more tissue-like features. Changes in overall gene expression was organized in main functional groups, concerning cell junctions, cell adhesion, cytochrome P450 metabolism, cell cycle, DNA mismatch repair and DNA replication. While cell adhesion and cell junction gene expression increased, DNA replication/cell cycle genes as well as base excision DNA repair genes expression decreased in $\mathrm{HLaC} 78$ cell line. These principal observations roughly conform with a study on prostate cancer in 2007 (16), with respect to cell cycling, cell adhesion and DNA repair genes. In contrast, no angiogenesis genes were upregulated in $\mathrm{HLaC} 78$ upon spheroid aggregation, and vascular endothelial growth factor was slightly downregulated. It has to be considered, however, that in older spheroids an upregulation of angiogenesis genes may also occur, when central hypoxia increases (in the present study HIF-1 was upregulated only non-significantly after 72 h). To transfer data obtained from HLaC78 expression analysis line and to determine key changes in switching between $2 \mathrm{D}$ and $3 \mathrm{D}$ conditions, the expression of selected 
Table IV. Log fold-changes in gene expression of selected genes in spheroids vs. monolayer cultures of HNSCC cell lines.

\begin{tabular}{|c|c|c|c|c|c|c|c|c|c|}
\hline & GJB6 & $\mathrm{CDH} 1$ & AKR1C1 & CYP1A1 & CYP1B1 & MKI & FEN1 & MLH1 & $\mathrm{RCF} 3$ \\
\hline \multicolumn{10}{|c|}{ Tight spheroids } \\
\hline HLaC78 & 3.06 & 2.17 & 4.64 & 2.83 & 1.86 & -5.55 & -1.42 & -0.37 & -3.30 \\
\hline $\mathrm{HSmC78}$ & 5.93 & 3.04 & 6.58 & 5.02 & 5.23 & -3.71 & 0.73 & 0.81 & -0.95 \\
\hline Cal27 & 5.05 & 1.81 & 4.04 & 4.69 & 2.55 & -1.17 & -0.22 & 1.34 & -0.61 \\
\hline PE/CA-PJ4I & 0.49 & 1.35 & 2.76 & 6.78 & 4.88 & -4.35 & 1.51 & -0.04 & -0.65 \\
\hline HNO210 & 5.75 & -0.44 & 5.99 & 1.49 & 0.12 & -6.48 & 3.31 & 0.40 & -2.65 \\
\hline SCC4 & 0.59 & 0.79 & 0.15 & 5.71 & 0.75 & -2.41 & -0.92 & 0.91 & -7.64 \\
\hline \multicolumn{10}{|c|}{ Loose/intermediate aggregates } \\
\hline$F A D U$ & -0.68 & 0.06 & 0.04 & 0.45 & 0.79 & 0.43 & -0.38 & -0.52 & -0.22 \\
\hline Нер2 & -2.52 & -1.62 & -0.77 & 1.21 & -2.21 & -0.48 & -1.85 & -0.68 & -1.57 \\
\hline Hep2-Tax & -2.23 & -2.20 & 2.36 & 1.26 & 0.07 & -1.44 & -1.71 & -3.17 & -0.65 \\
\hline $\mathrm{HPaC79}$ & -1.16 & -1.06 & -1.27 & -1.47 & -3.89 & -4.02 & -1.56 & -1.40 & -5.58 \\
\hline HLaC79 & -0.29 & 1.04 & 0.90 & 0.11 & 0.15 & -0.13 & -0.34 & -1.09 & -0.55 \\
\hline HLaC79-Tax & -5.22 & -3.24 & -7.64 & -2.55 & -0.99 & -0.01 & -5.95 & -0.28 & -0.68 \\
\hline
\end{tabular}

HNSCC, head and neck cancer.

genes of each functional group were analyzed by quantitative RT-PCR in monolayer and spheroid cultures of different HNSCC cell lines.

The decrease in cell cycling/DNA synthesis seemed to be a common mechanism also in HNSCC cell lines, as shown by the expression analysis of Ki67 and RCF3. Cell junction and adhesion gene upregulation, however, was restricted to those cell lines with distinct E-cadherin expression already under two-dimensional conditions. These cell lines usually experienced a further upregulation of E-cadherin upon spheroid aggregation. Cell lines with low E-cadherin levels in monolayer cultures, however, further decreased expression under 3D conditions. This is somewhat divergent from the findings of Kim et al (17), who unveiled E-cadherin directly regulating the hippo signaling pathway controlling cell proliferation. E-cadherin mediates contact inhibition, which is essential for tissue maintenance. In the cell lines producing only loose aggregates, proliferation decreased despite downregulation of E-cadherin, indicating further pathways, regulating proliferation under tissue-like conditions.

Upregulation of cytochrome P450 metabolism genes has been solely reported several times for hepatocellular carcinoma cell lines. Thus, overall gene expression was analysed in HepG2 liver carcinoma monolayer cells and corresponding spheroids (18). The authors reported upregulation of cytochrome p450 1A1 (CYP1A1) as well as aldo-keto reductase $\mathrm{C} 1$ (AKR1C1), both factors involved in cytochrome P450-mediated metabolism of xenobiotics in spheroids. Hsiao et al (19) showed that CYP1A1 and CYP1B1 activity increased in hepatocytes with assembly of spheroids and decreased with disassembly of spheroids on collagen coated surfaces.

Notably, Masood et al (20) reported downregulation of CYP1A1 mRNA in head and neck cancer specimens compared to normal mucosal tissue. Upregulation of the cytochrome
P450 enzymes or AKR1A1 in spheroids may therefore reflect a regression to a more regulated cell type in a tissue-like context.

Francia et al (21) performed a differential display analysis of monolayers and spheroids of EMT- 6 mammary carcinoma cells and compared differentially expressed genes with EMT-6 tumours selected in vivo to be resistant to alkylating agents. They identified the DNA mismatch repair-associated mismatch repair endonuclease 2 (PMS2) gene to be associated with resistance. Downregulation of PMS2 caused a multicellular resistance of tumour cell spheroids against alkylating agents. They hypothesized, that a deficiency in DNA mismatch repair may explain some forms of multicellular resistance, particularly to alkylating agents.

In HLaC78 genes of the functional group, DNA mismatch repair was significantly downregulated. We have not tested resistance of monolayers and spheroids of our cell lines against alkylating agents. Furthermore, it seems, that downregulation of DNA mismatch-repair genes is not a general mechanism of HNSCC cancer cell lines upon aggregation into spheroids, but it is notable, that the tested genes where more commonly downregulated in those cell lines forming loose clusters instead of tight spheroids.

Summarizing our results, it seems that in head and neck cancer cell lines (HNSCC) the 3D context reverses monolayer cells to growth and behavior of cells in a more tissue-like controlled condition with enhanced cell-cell adhesion and decreased proliferation. The formation of tight regular spheroids is dependent on distinct E-cadherin expression levels in monolayer cultures, usually resulting in further upregulation upon aggregation into 3D structures. Cell lines expressing only low levels of E-cadherin produce only loose cell clusters, frequently decreasing E-cadherin expression further. These cell lines diminish proliferation despite decreased E-cadherin. Common shifts in gene expression between 2D and 3D growth of HNSCC cell lines include the functional groups cell 
junctions, cell adhesion, cell cycle, DNA replication as well as genes concerning the metabolism of xenobiotics.

\section{Acknowledgements}

We would like to thank Dr Piet Tas $(\mathrm{PhD})$ for critical reading of the manuscript.

\section{References}

1. Guntinas-Lichius O, Wendt T, Buentzel J, Esser D, Lochner P, Mueller A, Schultze-Mosgau S and Altendorf-Hofmann A: Head and neck cancer in Germany: A site-specific analysis of survival of the Thuringian cancer registration database. J Cancer Res Clin Oncol 136: 55-63, 2010.

2. Griffith LG and Swartz MA: Capturing complex 3D tissue physiology in vitro. Nat Rev Mol Cell Biol 7: 211-224, 2006.

3. Wustrow TP, Raffael A and Valet G: Multiparametric flow cytometry of human squamous cell carcinoma lines from the head and neck. Otolaryngol Head Neck Surg 98: 552-557, 1988.

4. Lin RZ and Chang HY: Recent advances in three-dimensional multicellular spheroid culture for biomedical research. Biotechno J 3: 1172-1184, 2008.

5. Zenner HPLW, Lehner W and Herrmann IF: Establishment of carcinoma cell lines from larynx and submandibular gland. Arch Otorhinolaryngol 225: 269-277, 1979.

6. Zenner HP, Herrmann IF, Bremer W and Stahl-Maugé C: Head and neck carcinoma models. In vivo reproduction in athymic mice and in vitro culture. Acta Otolaryngol 95: 371-381, 1983.

7. Berndt A, Hyckel P, Könneker A, Katenkamp D and Kosmehl H: Oral squamous cell carcinoma invasion is associated with a laminin-5 matrix re-organization but independent of basement membrane and hemidesmosome formation. Clues from an in vitro invasion model. Invasion Metastasis 17: 251-258, 1997.

8. Vinci M, Gowan S, Boxall F, Patterson L, Zimmermann M, Court W, Lomas C, Mendiola M, Hardisson D and Eccles SA: Advances in establishment and analysis of three-dimensional tumor spheroid-based functional assays for target validation and drug evaluation. BMC Biol 10: 29, 2012.

9. Irizarry RA, Hobbs B, Collin F, Beazer-Barclay YD, Antonellis KJ, Scherf U and Speed TP: Exploration, normalization, and summaries of high density oligonucleotide array probe level data. Biostatistics 4: 249-264, 2003.
10. Bolstad BM, Irizarry RA, Astrand M and Speed TP: A comparison of normalization methods for high density oligonucleotide array data based on variance and bias. Bioinformatics 19: 185-193, 2003.

11. Subramanian A, Tamayo P, Mootha VK, Mukherjee S, Ebert BL, Gillette MA, Paulovich A, Pomeroy SL, Golub TR, Lander ES, et al: Gene set enrichment analysis: A knowledge-based approach for interpreting genome-wide expression profiles. Proc Natl Acad Sci USA 102: 15545-15550, 2005.

12. Livak KJ and Schmittgen TD: Analysis of relative gene expression data using real-time quantitative PCR and the $2-^{\Delta \Delta C T}$ method. Methods 25: 402-408, 2001.

13. Stockinger A, Eger A, Wolf J, Beug H and Foisner R: E-cadherin regulates cell growth by modulating proliferation-dependent beta-catenin transcriptional activity. J Cell Biol 154: 1185-1196, 2001.

14. Wang D, Su L, Huang D, Zhang H, Shin DM and Chen ZG: Downregulation of E-cadherin enhances proliferation of head and neck cancer through transcriptional regulation of EGFR. Mol Cancer 10: 116, 2011 .

15. Ivascu A and Kubbies M: Diversity of cell-mediated adhesions in breast cancer spheroids. Int J Oncol 31: 1403-1413, 2007.

16. Takagi A, Watanabe M, Ishii Y, Morita J, Hirokawa Y, Matsuzaki T and Shiraishi T: Three-dimensional cellular spheroid formation provides human prostate tumor cells with tissue-like features. Anticancer Res 27: 45-53, 2007.

17. Kim NG, Koh E, Chen X and Gumbiner BM: E-cadherin mediates contact inhibition of proliferation through Hippo signaling-pathway components. Proc Natl Acad Sci USA 108: 11930-11935, 2011

18. Chang TT and Hughes-Fulford M: Monolayer and spheroid culture of human liver hepatocellular carcinoma cell line cells demonstrate distinct global gene expression patterns and functional phenotypes. Tissue Eng Part A 15: 559-567, 2009.

19. Hsiao CC, Wu JR, Wu FJ, Ko WJ, Remmel RP and Hu WS Receding cytochrome $\mathrm{P} 450$ activity in disassembling hepatocyte spheroids. Tissue Eng 5: 207-221, 1999.

20. Masood N, Malik FA and Kayani MA: Expression of xenobiotic metabolizing genes in head and neck cancer tissues. Asian Pac J Cancer Prev 12: 377-382, 2011.

21. Francia G, Man S, Teicher B, Grasso L and Kerbel RS: Gene expression analysis of tumor spheroids reveals a role for suppressed DNA mismatch repair in multicellular resistance to alkylating agents. Mol Cell Biol 24: 6837-6849, 2004. 